\title{
Glucocorticoid therapy for trauma - ready for prime time?
}

\author{
Venkata S Aleti and Sachin Yende* \\ University of Pittsburgh Department of Critical Care Medicine: Evidence-Based Medicine Journal Club, edited by Sachin Yende
}

\section{Expanded abstract Citation}

Roquilly A, Mahe PJ, Seguin P, et al:: Hydrocortisone Therapy for Patients with Multiple Trauma: The Randomized Controlled HYPOLYTE Study. JAMA 2011, 305: 1201-1209.

Clinicaltrials.gov Identifier: NCT00563303

\section{Background}

The role of stress-dose hydrocortisone in the management of trauma patients is currently unknown.

\section{Methods}

Objective: To test the efficacy of hydrocortisone therapy in trauma patients.

Design: Multicenter randomized, double blind, placebocontrolled study.

Setting: Seven intensive care units (ICUs) in France during November 2006 to August 2009.

Subjects: 150 patients with severe trauma who required ICU stay for at least 48 hours were included in the study.

Intervention: Patients were randomly assigned to a continuous intravenous infusion of either hydrocortisone (200 mg/day for 5 days, followed by $100 \mathrm{mg}$ on day 6 and $50 \mathrm{mg}$ on day 7) or placebo. The treatment was stopped if patients had an appropriate adrenal response.

Outcomes: Hospital-acquired pneumonia within 28 days. Secondary outcomes included the duration of mechanical ventilation, ICU length of stay, hyponatremia, and death.

\section{Results}

An intention-to-treat (ITT) analysis included 149 patients and the modified ITT analysis included 113 patients with corticosteroid insufficiency. In the ITT analysis, 26 of 73 patients $(35.6 \%)$ treated with hydrocortisone and 39 of 76 patients (51.3\%) receiving placebo

*Correspondence: yendes@upmc.edu

Department of Critical Care Medicine, University of Pittsburgh, 606D Scaife Hall, 3550 Terrace Street, Pittsburgh, PA 15261, USA developed hospital-acquired pneumonia by day 28 (hazard ratio (HR), 0.51; 95\% confidence interval (CI), $0.30-0.83 ; P=0.007)$. In the modified ITT analysis, 20 of 56 patients (35.7\%) in the hydrocortisone group and 31 of 57 patients $(54.4 \%)$ in the placebo group developed hospital-acquired pneumonia by day 28 (HR, 0.47; 95\% CI, 0.25-0.86; $P=0.01$ ). Mechanical ventilation-free days increased with hydrocortisone use by 4 days (95\% CI, 2-7; $P=0.001)$ in the ITT analysis and 6 days (95\% CI, 2-11; $P<0.001)$ in the modified ITT analysis. Hyponatremia was observed in 7 of $76(9.2 \%)$ in the placebo group vs. none in the hydrocortisone group (absolute difference, $-9 \%$; $95 \% \mathrm{CI},-16 \%$ to $-3 \% ; P=0.01)$. Four of 76 patients $(5.3 \%)$ in the placebo group and 6 of $73(8.2 \%)$ in the hydrocortisone group died (absolute difference, 3\%; 95\% $\mathrm{CI},-5 \%$ to $11 \% ; P=0.44)$.

\section{Conclusions}

In intubated trauma patients, the use of an intravenous stress-dose of hydrocortisone, compared with placebo, resulted in a decreased risk of hospital-acquired pneumonia.

\section{Commentary}

Severe trauma is a leading cause of death, especially in younger adults. Patients who survive the initial trauma are at high risk for infections, which often increases subsequent morbidity and mortality. Pneumonia is a common infection, occurring in $40-60 \%$ of trauma patients. Shock and resuscitation following trauma may lead to an exaggerated immune response [1]. The "two hit hypothesis" states that an initial less severely injured patient may eventually develop worsening of multi-organ failure as a result of reactivation of their inflammatory response due to minor intercurrent event [2], such as an infection. Thus, preventing infection in trauma patients is important.

The effect of glucocorticoid therapy on susceptibility to infections is controversial. Several lines of evidence suggest that glucocorticoid therapy may reduce risk of infection. For example, higher circulating levels of proinflammatory cytokines, such as tumor necrosis factor (TNF) and interleukin (IL)-6, may increase risk of 
infection in animal models and human studies [3,4]. Glucocorticoids inhibit nuclear factor- $\mathrm{kB}$ and transcription of genes that code for pro-inflammatory cytokines, including TNF, IL-1, IL-6, IL-8, and interferon (IFN) $-\gamma$ $[5,6]$, and may therefore prevent infection. The acute proinflammatory response following trauma is followed by a compensatory anti-inflammatory response, where immune cells may experience tolerance (immunoparalysis). Although immunoparalysis may be important to dampen the deleterious effects of the pro-inflammatory response, patients may be at higher risk for secondary infections during this phase. By reducing the early inflammatory response, glucocorticoids may reduce the magnitude of the subsequent anti-inflammatory response, thereby reducing risk of secondary infections. However, some studies suggest that glucocorticoid therapy could have deleterious effects on infection risk. For instance, the risk of secondary infections was higher in patients who received glucocorticoid therapy in the CORTICUS [7] trial. No large randomized clinical trial has examined the efficacy of glucocorticoids to prevent infections and the current study by Roquilly et al. remains one of the largest studies to test this hypothesis.

In this study the author enrolled 150 patients with an injury severity score of 15 and above and included patients with traumatic brain injury (managed at a level 1 trauma center) and randomized them to cortisol and placebo treated arms. Baseline cortisol was measured and patients who were cortisol-deficient were given steroid treatment for 1 week. The author determined occurrence of pneumonia within 28 days of hospitalization and found that patient who received steroid treatment had a significantly lower incidence of pneumonia compared to placebo (HR, $0.51 ; P=0.007$ ).

The results of this study are in contrast with the results of the CRASH [8] study. The CRASH study was a large multicenter randomized clinical trial conducted in 10,000 patients with head injury, as evidenced by Glasgow Coma Score of 14 or less, to determine whether high dose glucocorticoids improve outcomes. However, there were important differences between the HYPOLYTE and CRASH studies (Table 1). In contrast to CRASH, which focused on traumatic head injury patients alone, HYPOLYTE enrolled a broad population of patients with trauma and included a smaller subset (44.96\%) with head injury. Both dose and duration of glucocorticoid therapy were different. CRASH used high dose steroids, which may lead to immunosuppression. HYPOLYTE used lowmoderate dose glucocorticoids, which are less likely to cause immune suppression and may be important in patients who have critical illness-related corticosteroid insufficiency (CIRCI). The CRASH study treated all patients with glucocorticoid therapy, whereas the HYPOLYTE study discontinued therapy in those who did
Table 1. Differences between HYPOLYTE and CRASH studies

\begin{tabular}{lcc}
\hline & CRASH STUDY & HYPOLYTE STUDY \\
\hline $\begin{array}{l}\text { Number of patients } \\
\text { Primary end point }\end{array}$ & 10,000 & 150 \\
$\begin{array}{l}\text { Time to administration of } \\
\text { first dose of steroid }\end{array}$ & $<8$ hours & $\begin{array}{c}\text { Within } 36 \text { hours of } \\
\text { trauma; } 25 \text { hours } \\
\text { (median) }\end{array}$ \\
$\begin{array}{l}\text { Steroid loading dose } \\
\text { Duration of Steroids }\end{array}$ & 2 gms & None \\
\hline
\end{tabular}

not meet criteria for relative adrenal insufficiency. Duration of therapy also differed between these studies and shorter course of steroids can be associated with rebound inflammation. Finally, the primary endpoint in these studies was also different. For example, the primary endpoint was all-cause mortality at 2 weeks in the CRASH study, whereas the HYPOLYTE study examined effect of steroids on hospital-acquired pneumonia. The CRASH study was stopped early due to higher mortality in the steroid treated arm, whereas the current study showed no difference in mortality between the two treatment groups.

This study by Roquilly et al. has several limitations. First, cortisol levels drawn prior to administering steroids may be influenced by the prior use of etomidate for intubation, $(63 \%$ of patients in this study received etomidate prior to intubation), which can cause adrenal suppression for approximately 24 hours $[9,10]$. Thus, the high rate of adrenal insufficiency may be influenced by etomidate administration. Second, it is unclear whether steroid therapy should be targeted based on presence or absence of relative adrenal insufficiency. Although the corticotropin stimulation test is recommended to diagnose CIRCI, results of the CORTICUS trial showed no difference in the efficacy of glucocorticoid therapy among those with and without relative adrenal insufficiency. Third, the investigators did not assess the longterm effects of steroid use, such as myopathy and critical illness neuropathy, a potential complication of glucocorticoid therapy. Finally, the study did not determine mechanisms of beneficial effects of steroids. For example, whether steroids reduced risk of infection by reducing pro-inflammatory markers or improved immune cell function is not known.

\section{Recommendation}

Although results of this study are intriguing, glucocorticoids cannot be recommended for routine use in trauma patients. These results suggest a need to conduct a larger trial to understand whether a reduction in infection observed in this study translates into improved mortality. 


\section{Abbreviations}

$\mathrm{Cl}$, confidence interval; $\mathrm{CIRCl}$, critical illness-related corticosteroid insufficiency: HR, hazard ratio; ICU, intensive care unit; IFN, interferon; IL, interleukin; ITT, intention-to-treat; TNF, tumor necrosis factor;

\section{Competing interests}

The authors declare that they have no competing interests.

Published: 20 January 2012

\section{References}

1. Rotstein OD: Modeling the two-hit hypothesis for evaluating strategies to prevent organ injury after shock/resuscitation. J Trauma 2003, 54:S203-S206.

2. Saadia R, Schein M: Multiple organ failure. How valid is the "two hit" model? J Accid Emerg Med 1999, 16:163-166.

3. Hinojosa E, Boyd AR, Orihuela CJ: Age-associated inflammation and toll-like receptor dysfunction prime the lungs for pneumococcal pneumonia. $J$ Infect Dis 2009, 200:546-554

4. Yende $\mathrm{S}$, Tuomanen El, Wunderink R, et al: Preinfection systemic inflammatory markers and risk of hospitalization due to pneumonia. Am J Respir Crit Care Med 2005, 172:1440-1446.

5. Rhen T, Cidlowski JA: Antiinflammatory action of glucocorticoids - new mechanisms for old drugs. N Eng/ J Med 2005, 353:1711-1723.
6. Confalonieri M, Urbino R, Potena A, et al.: Hydrocortisone infusion for severe community-acquired pneumonia: a preliminary randomized study. Am J Respir Crit Care Med 2005, 171:242-248.

7. Sprung CL, Annane D, Keh D, et al:: Hydrocortisone therapy for patients with septic shock. N Engl J Med 2008, 358:111-124.

8. Roberts I, Yates D, Sandercock P, et al.: Effect of intravenous corticosteroids on death within 14 days in 10008 adults with clinically significant head injury (MRC CRASH trial): randomised placebo-controlled trial. Lancet 2004, 364:1321-1328

9. de Jong FH, Mallios C, Jansen C, Scheck PA, Lamberts SW: Etomidate suppresses adrenocortical function by inhibition of 11 beta-hydroxylation. J Clin Endocrinol Metab 1984, 59:1143-1147.

10. Marik PE, Pastores SM, Annane D, et al.: Recommendations for the diagnosis and management of corticosteroid insufficiency in critically ill adult patients: consensus statements from an international task force by the American College of Critical Care Medicine. Crit Care Med 2008 , 36:1937-1949.

doi:10.1186/cc10596

Cite this article as: Aleti VS, Yende S: Glucocorticoid therapy for trauma ready for prime time? Critical Care 2012, 16:301. 